\title{
Investigación-acción para conseguir grupos colaborativos eficaces
}

\section{Action research to get effective collaborative groups}

\author{
María José Labrador-PIQuer ${ }^{1}$ \\ mlabrado@upvnet.upv.es \\ María Ángeles Andreu-Andrés \\ maandreu@idm.upv.es \\ Universitat Politècnica de València, España
}

\section{Resumen:}

Tras un breve recorrido por los orígenes de la investigación-acción, en línea con la práctica reflexiva del profesor investigador, este estudio plantea las dificultades encontradas en dos grupos de estudiantes que presentan unos resultados ineficaces de trabajo grupal y describe la técnica colaborativa empleada en la experiencia para lograr unos resultados satisfactorios, el puzle de Aronson. Tras su implementación se recogen los resultados y conclusiones obtenidas a través de un análisis cualitativo (para estudiar la percepción del alumno) y uno cuantitativo (para analizar el número de miembros de los grupos de trabajo que desarrollan sus tareas adecuadamente). Estos resultados pueden ayudar a los docentes a reflexionar y realizar los cambios necesarios en estas y en otras situaciones educativas.

\section{Palabras clave:}

Investigación-acción; trabajo en grupo; puzle de Aronson; percepciones de los estudiantes; análisis cualitativo; análisis cuantitativo.

\begin{abstract}
:
This research study briefly reviews the origins of action-research in accordance with the reflective practice of lecturers-researchers; it sets out the difficulties found in two groups of students who obtained poor results when working in groups and describes the collaborative technique implemented to improve such a behavior, Aronson's jigsaw. After working with this technique our study analyses the students' perceptions by means of a qualitative analysis. A quantitative analysis of the number of students per team and the number of those who did their tasks adequately offer complementary results that can help lecturers reflect on the experience and fulfill any necessary changes in current and future educational settings.
\end{abstract}

\section{Key words:}

Action-research; teamwork; Aronson's jigsaw; students' perceptions; qualitative analysis; quantitative analysis.

1 Dirección para correspondencia (correspondence address):

María José Labrador-Piquer. Escuela Técnica Superior de Ingeniería Informática. Universitat Politècnica de València. Camino de Vera, s/n. 46022 Valencia (España). 


\section{Résumé:}

Après un bref parcours à travers les origines de la recherche-action, en accord avec l'exercice de réflexion du professeur chercheur, cet exposé établit les difficultés constatées avec des groupes d'étudiants qui présentent de mauvais résultats en ce qui concerne le travail en équipe et décrit la technique de collaboration employée durant l'expérience afin d'obtenir des résultats satisfaisants: le puzzle de Aranson. Après avoir appliqué cette méthode, on a recueilli des résultats et des conclusions obtenues par l'analyse qualitative (afin d'étudier la perception de l'élève) et une analyse quantitative (pour analyser le nombre de participants des groupes de travail qui réalisent leurs tâches de façon satisfaisante). Ces résultats peuvent aider les professeurs à réfléchir et à réaliser les changements nécessaires en lien avec leur activité éducative. Mots clés:

Recherche-action; travail en équipe; puzzle de Aranson; perception des étudiants; analyse qualitative; analyse quantitative.

Fecha de recepción: 26-4-2013

Fecha de aceptación: 8-4-2014

\section{Antecedentes}

De los once criterios especificados por la organización no gubernamental ABET (2000) en disciplinas de ingeniería y tecnología, Passow (2012) señala que - con pocas excepciones - los estudiantes de ingeniería graduados valoran con diferencia un grupo de competencias compuesto por la resolución de problemas, el análisis de datos, las destrezas comunicativas y el trabajo en grupo como las más importantes en su experiencia laboral. Sin embargo, al implementarlas en nuestro programa nos hemos encontrado con grupos de estudiantes que presentan resultados ineficaces de trabajo grupal que nos han llevado a poner en práctica la investigación-acción en el aula a fin de intentar corregir esos resultados.

Antes de adentrarnos en el objeto de estudio y la metodología seguida, centramos los siguientes subapartados en el marco teórico en el que este trabajo se apoya: la investigación-acción —a través de unas pinceladas que no pretenden ser exhaustivas dada la extensión de este campo-, la práctica reflexiva del profesor investigador y la técnica colaborativa implementada para paliar las dificultades encontradas en los grupos de trabajo. Una vez presentado el perfil del alumnado y el método de estudio y análisis empleado, se ofrecen los resultados de implementación a través de un análisis cualitativo y cuantitativo. 


\section{Marco teórico}

\section{Contextualización de la investigación-acción y la práctica docente}

Los orígenes de la investigación-acción, como nos indica Masters (1995), no están del todo claros. Algunos autores como Kemmis y McTaggart (1998) o Holter y Schwarts-Barcott (1993) los sitúan con Lewin; McKernan (1991, ed. 2010) señala que, como método, parece tener sus orígenes en el movimiento educativo de finales del siglo XIX y principios del XX en el que el método científico se aplica a la educación, así como en reformistas anteriores a Lewin que ya en 1913 utilizan la dinámica de grupos en psicología social. Autores como Beverly (1993) afirman que el concepto se remonta a los trabajos realizados por Dewey en 1920 y a los de Lewin, veinte años después. Sin embargo, subrayan que es Corey y otros profesores del Teachers College de la Universidad de Columbia quienes introducen el término ante la comunidad educativa en 1949. Otros, en cambio, como Brydon-Miller, Greenwood y Maguire (2003) señalan que situar sus orígenes es tarea realmente compleja al tratarse de un modo de hacer investigación que ha ido surgiendo con el tiempo desde campos de estudio muy diferenciados.

No obstante, puede afirmarse que a mediados de 1940 Lewin construye una teoría sobre la investigación-acción que reúne en una misma práctica la docencia y la investigación; consiste en una serie de decisiones (pasos en espiral) que constan de planificación, acción, observación y evaluación de los resultados de la actuación. Este autor defiende que los problemas sociales (o educativos) deben servir como centro de investigación al integrar elementos de la investigación experimental y las ciencias sociales. Se considera que es Lewin quien desarrolla y presenta por primera vez el concepto de investigación-acción en su artículo Action Research and Minority Problems publicado en 1946. Años más tarde, destacan los trabajos de Stenhouse $(2003,2009)$ en los que se sostiene que la enseñanza debe basarse en la investigación y tanto la investigación como el desarrollo del currículo han de preservar dicha enseñanza.

De manera tradicional, la comunidad en la que se realiza una investigación no se inmiscuye ni en el proceso investigador ni en sus resultados, sólo logra conocer las conclusiones a las que se llega. En las últimas décadas han surgido enfoques de investigación que buscan mayor parti- 
cipación del grupo o la comunidad en la que se lleva a cabo el estudio y que se beneficia directamente de él. La investigación-acción se sitúa entre estos enfoques y para autores como Elliot (1990, ed. 2010) es la forma de reflexión que más se suele dejar de lado, en comparación con las tradicionales. No obstante, la tendencia parece estar cambiando desde una investigación cuantitativa a otra que recibe diferentes nombres según los autores: profesor-investigador (Cochran-Smith y Little, 1993), práctica-reflexiva (Schön, 1983, 1987) o investigación-acción (Winter, 1987 ed. 2009; Carr, 1989), entre otros.

Schön (1983) destaca dos conceptos que considera esenciales respecto al modo en que el docente reflexiona sobre su propia enseñanza; los denomina reflexión durante y tras la acción. El concepto de práctica reflexiva gira en torno a la idea del aprendizaje a lo largo de la vida, en el que los participantes analizan experiencias a fin de aprender de ellas, y promueve profesionales independientes y reflexivos. Este proceso pasa por el diagnóstico de la situación actual en el aula, la identificación del problema o mejora que se desea implementar, la formulación de hipótesis posibles, la selección y el diseño de la acción y finalmente, la evaluación de sus efectos (Kemmis y McTaggart, 1988; Jiménez-Domínguez, 1994). Se trata de un modelo de investigación cíclica y reflexiva, útil para quienes quieren comprender mejor su práctica docente (Carr y Kemmis, 1986), que fomenta la calidad de la enseñanza.

Cochran-Smith y Lyttle (1993) matizan que el profesor-investigador toma diferentes formas y tiene diferentes propósitos; el docente en ejercicio es investigador de la acción, dirige la investigación de forma individual o colectiva con el principal objetivo de comprender la enseñanzaaprendizaje en contexto y desde la perspectiva de quienes interactúan día a día en el aula.

Winter (1987), por su parte, señala que el propósito de la investigación acción es proporcionar una teoría analítica de la investigaciónacción, un conjunto de condiciones para su posibilidad teórica. Su principal preocupación es el desarrollo de la autorreflexión y la dinámica del poder de reflexión en la investigación.

Desde el punto de vista metodológico, nuestro estudio gira en torno al modelo de enseñanza-aprendizaje colaborativo; un aprendizaje basado en el socioconstructivismo en el que el aprendizaje deja de ser considerado como la adquisición de conocimientos de un individuo para ser reconocido como un proceso de participación social. Hermana aspectos 
de la obra de Piaget, Bruner y Vygotsky en la que se expone el hecho de que todos adquirimos conocimiento y significado de nuestras experiencias al construirlas de manera activa, nunca del entorno pasivo que nos rodea o de los propios profesores sin más. Tiene en cuenta la sociedad que afecta a los propios estudiantes; por lo tanto, estrategias metodológicas que hacen uso de esta concepción de la enseñanza-aprendizaje como es el caso del aprendizaje colaborativo, y en concreto del puzle de Aronson que ponemos en práctica en esta investigación y del cual nos ocupamos en el siguiente apartado, sitúan a los participantes en un contexto con significado en el que el trabajo en pequeños grupos, la negociación y la discusión destaca frente a las respuestas correctas.

Como aprendizaje activo, una de las bases fundamentales del aprendizaje colaborativo, tiene presente la idea que Comenius (1592-1670) sustenta en su Didáctica Magna ${ }^{2}$ cuando invita a sus lectores a encontrar un método en el que los profesores gasten menos tiempo enseñando y los estudiantes aprendan más al emplear menos tiempo escuchando de manera pasiva.

\section{El profesor como investigador y profesional}

Durante siglos la enseñanza y la investigación han coexistido en educación como actividades diferenciadas - tal y como ha ocurrido con la teoría y la práctica- (Woods, 1986; Slavin 1990; Stenhouse, 2003) si bien, en la actualidad, convive esa postura junto con la que defiende que se encuentren integradas, especialmente si se entiende la enseñanza como una actividad autorreflexiva (Latorre, 2003; Sim y Radolff, 2008; Burns, 2010). Según Latorre (2003, ed. 2010), esta idea va tomando fuerza en el ámbito universitario con un profesional que se formula preguntas sobre su docencia, recoge datos en el aula, los analiza, interpreta y, si cabe, se plantea nuevas preguntas/hipótesis fruto de las propias experiencias de aula (Bartolomé Pina, 1992; López Górriz, 1993; Elliot, 1993; Rincón y Rincón, 2000).

La investigación-acción generalmente no se ocupa de problemas teóricos de un área de conocimiento concreta, sino de problemas prácticos relacionados con el quehacer docente (Aranguren, 2007). La investigación-acción que se ha realizado en este estudio ha sido en equipo y ha

2 http://core.roehampton.ac.uk/digital/froarc/comgre/\#intros y http://core.roehampton. ac.uk/digital/froarc/comgre/part2/fly2.pdf 
proporcionado la posibilidad de compartir dudas, preocupaciones o resultados que ayudan a prevenir el aislamiento del docente y facilitan el diálogo entre profesionales. Entre otros ejemplos se encuentran los proyectos multidisciplinares de Casals et al. (2008) o los de Quintero et al. (2003) que estudian mejoras en la organización y el desarrollo de las clases.

Para llevarla a cabo, se parte del análisis de la realidad del aula (qué está sucediendo), lo que conlleva observación, diagnóstico y actuación; como respuesta se realiza un programa de acción seguido de su implementación. Posteriormente, tras la observación y recogida de datos de los efectos producidos, se analizan y se plantean posibles modificaciones o la planificación de un nuevo proceso. La observación y la recogida y análisis de datos de manera rigurosa es lo que le confiere rango de investigación (véase figura 1).

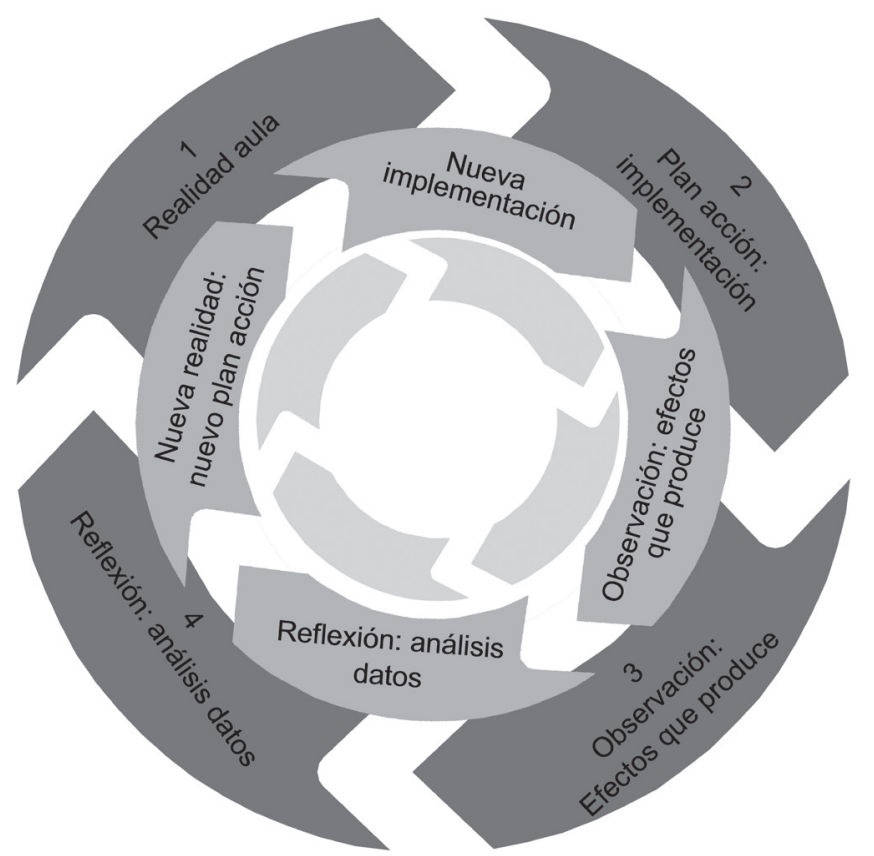

Figura 1. Ciclo de la investigación-acción (elaboración propia)

\section{Hacia el puzle de Aronson como técnica de aprendizaje colaborativo}

Los estudios sobre aprendizaje colaborativo se han centrado fundamentalmente en educación Primaria y Secundaria; solo en los últimos años se está investigando en niveles universitarios (Echazarreta y otros, 2009; Pé- 
rez-Sánchez, Poveda-Serra y Gilar-Corbí, 2010). Existen otras expresiones para designar este tipo de actividad y no se suele hacer ninguna diferenciación, fundamentalmente en los diccionarios ${ }^{3}$ : aprendizaje en grupo, en equipo, cooperativo o colaborativo; sin embargo, algunos autores señalan diferencias entre colaborativo y cooperativo (Cuseo, 1992; Bruffee, 1993; Flannery, 1994) e indican que en el aprendizaje cooperativo el profesor sigue siendo el experto y la autoridad en el aula, mientras que en el colaborativo profesor y alumno trabajan juntos para crear el saber. Bruffee (1993), defensor de tales distinciones, afirma que el saber es algo que construyen las personas hablando entre ellas y poniéndose de acuerdo; de este modo, el profesor - tal y como argumenta Mattews, 1996 - se convierte, junto con los alumnos, en una comunidad que busca el saber.

Trabajar de forma colaborativa no es un fin en sí mismo sino un instrumento complementario de mejora puesto que no se aprende espontáneamente sino que requiere una preparación, una organización meticulosa y un aprendizaje. No se trata de pedir a los estudiantes que se reúnan en grupos y trabajen sin más, si no de que cada integrante tenga una razón para tomarse en serio el rendimiento de los demás compañeros de grupo. Su pedagogía parte de la base de que los individuos crean significados al colaborar y el proceso les enriquece y les hace crecer.

En el aprendizaje colaborativo los docentes estructuran las tareas de aprendizaje intencional, en parejas o en grupos, de manera que comparten equitativamente la carga y responsabilidad de trabajo a la par que progresan hacia los resultados de aprendizaje previstos (Barkley, Cross y Major, 2009). Su meta es el desarrollo de personas reflexivas y autónomas y se considera más apropiado para estudiantes universitarios frente a otros niveles educativos. Otros investigadores, según McKernan (1996), como los socioetnógrafos Hammersley y Atkinson (1983), etnógrafos educativos como Goetz y Le Compte (1984) entre otros, utilizan las notas de campo detalladas como herramienta científica fundamental. En este enfoque el trabajo no está estructurado de antemano y el investigador toma nota de las cosas como son y no como están programadas; de esta forma no solo registra la conducta sino que participa en esa conducta. Como puede apreciarse, investigación-acción hoy en día no encaja en un enfoque estable, como ya señalaba McKernan (1996), pero se reconocen diversos estilos de investigación del curriculo, desde el trabajo racional del estudio de casos a la labor del profesor-investigador.

3 Por ejemplo el DRAE. 
En este estudio, dentro del grupo de trabajo colaborativo, consideramos que debe darse una interdependencia positiva entre sus miembros de modo que se sientan motivados para ayudarse a fin de conseguir los objetivos. Debe existir una responsabilidad tanto individual como grupal por lo que cada uno debe comprometerse a realizar su parte del trabajo para favorecer a los compañeros. Alentadas por estos conceptos emprendimos la implementación de la técnica y nos planteamos cómo organizar los grupos.

Dependiendo de su duración y finalidad los grupos de aprendizaje pueden ser informales, formales o básicos. Para Johnson y otros (1991, ed. 2009) un grupo informal se constituye aleatoriamente y se mantiene durante una actividad o parte de una clase. El grupo formal puede durar una sola sesión de clase o varias semanas, según el tiempo que precise la tarea colaborativa; su composición no es casual y persigue objetivos más complejos. Por el contrario, el grupo básico permanece durante todo un cuatrimestre o incluso un curso académico. La técnica del puzle, en particular, exige un grupo formal hasta la finalización de la tarea y las funciones del profesor son esenciales para su correcta implementación; de ahí que sea aconsejable para el docente una formación en dinámica de grupos (si no se tuviere) antes de comenzar el aprendizaje colaborativo en el aula (Labrador, 2011).

No existe una opinión única respecto al papel que debe desempeñar el docente a la hora de crear un ambiente de aprendizaje colaborativo aunque, por lo general, el papel que adopte suele ser reflejo de su estilo docente y de las necesidades de los equipos. De entre los cinco posibles estilos identificados por Grasha (2002) optamos en nuestra experiencia por el papel fundamental de facilitador que diseña la experiencia y el de observador, entre otros.

Consideramos que una experiencia de trabajo colaborativo con el puzle de Aronson no es aconsejable realizarla al principio de curso o cuando el docente desconoce si los estudiantes están familiarizados con el trabajo en equipo. En su lugar, es conveniente ir realizando de manera progresiva actividades en parejas y pequeños grupos, de modo que tanto discentes como docente se habitúen a la dinámica antes de poner en marcha la técnica. En la página web del Ministerio de Educación espa$\tilde{n}^{1}{ }^{4}$ se pueden encontrar diferentes ejemplos orientativos.

4 Ministerio de Educación. Instituto de Tecnologías Educativas - Acción tutorial - Dinámica de grupos http://ntic.educacion.es/w3//recursos2/orientacion/03accion/op03.htm 
La técnica Jigsaw fue diseñada por Aronson en 1978 y consiste en formar equipos denominados grupos puzle. Cada uno de sus miembros se hace responsable de una parte diferente de la tarea y, con ello, se convierte en experto de esa parte; todos los expertos en la misma tarea o subtema se constituyen en un grupo denominado grupo de expertos. Una vez elaborado el subtema, los miembros del grupo de expertos vuelven a su grupo puzle para exponerlo y recibir información del resto de subtemas por parte de sus compañeros. De este modo, la totalidad del trabajo está condicionado por la colaboración y la responsabilidad mutua entre sus miembros (véase figura 2). Los estudiantes aprenden, por una parte, el hecho de que nadie puede lograr nada positivo sin la ayuda de los otros y, por otra, la realidad de que cada miembro contribuye personalmente al trabajo de todos (Traver y García, 2006).

Entre las críticas que se realizan a la técnica destaca la de que el profesor está prácticamente ausente ${ }^{5}$, por ello algunos autores recomiendan llamar al grupo de expertos compañero-profesor. De igual modo, cabe plantearse la posible pérdida de control del docente sobre la información y el posible deterioro de su transmisión durante el uso de la técnica. Sin embargo, autores como Dansereau (1988) han demostrado que cuando se da una buena interdependencia positiva entre compañeros y una cierta autonomía en la captación de contenidos, el rendimiento del alumnado no sólo no disminuye sino que además fomenta la adquisición de destrezas que le permite la búsqueda, estructuración y transmisión de esa información a sus propios compañeros.

La fase final de evaluación del producto debe ser individual; en ella los alumnos informan (oralmente o por escrito) sobre los contenidos aprendidos de los compañeros y contestan a las preguntas abiertas que se formulen bien por escrito, en debate general o dentro de cada grupo.

5 Opinión que no compartimos ya que el profesor desempeña diferentes tareas: observador participante u orientador cuando es necesario. 


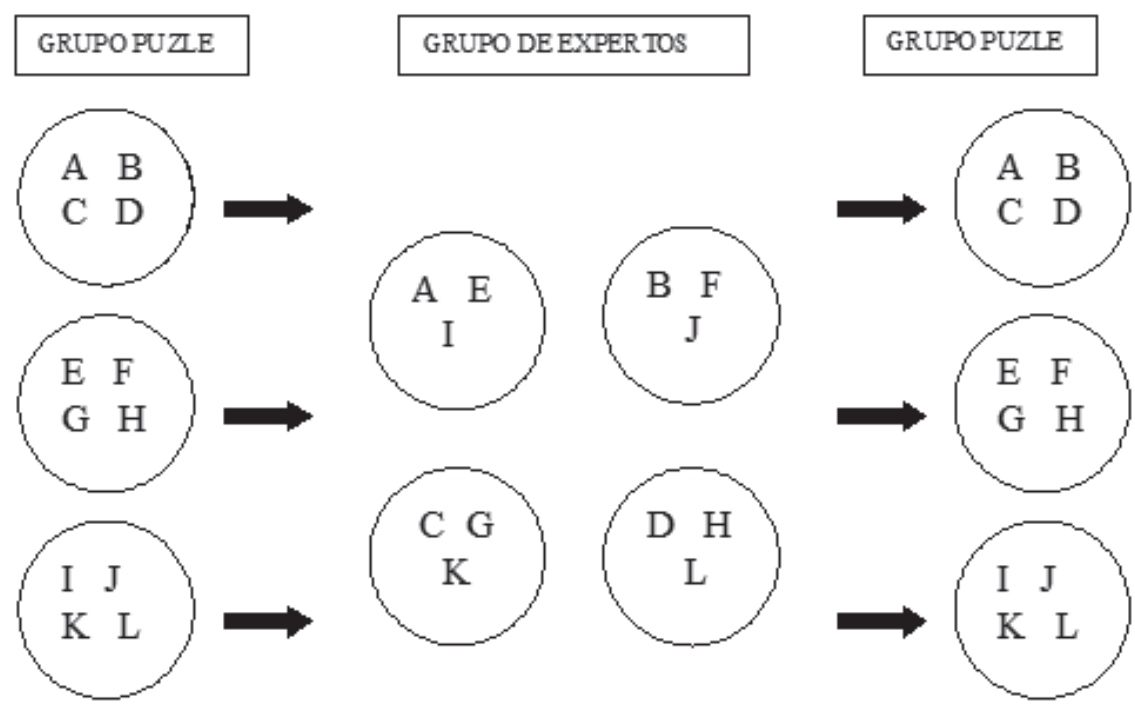

Figura 2. Diagrama fases del puzle de Aronson (Labrador et al., 2009)

Estudios sobre el empleo de esta técnica en el aula de lengua se centran en la enseñanza de géneros literarios (Gocer, 2010), la expresión escrita (Englert et al., 2001), o la eficacia de la técnica en la producción oral (Ghaith, 2004), entre otros.

\section{Objetivos de la investigación y perfil de los estudiantes}

El objetivo de este estudio no se centra en la defensa del método ideal sino que, siguiendo la espiral del proceso de investigación-acción, persigue la mejora de una situación insatisfactoria que se produce en el aula en unos grupos donde desempeñan su labor dos profesores diferentes. Los estudiantes no trabajan de forma eficiente, entre otros motivos, porque las aportaciones de los miembros de los grupos son muy dispares, se producen ausencias, no terminan las tareas $y$, consecuentemente, se desmotivan. De ahí que se planteen las siguientes preguntas: ¿Cómo conseguir que los alumnos finalicen sus tareas? ¿Cómo fomentar la responsabilidad en el grupo? ¿Cómo solucionar el problema de las ausencias? Ante esta situación se decide elegir una técnica colaborativa que ayude a solucionar el problema y de la que nos hemos ocupado en el apartado anterior. 
Cuatro objetivos animan esta propuesta; en primer lugar, que los estudiantes reflexionen sobre el trabajo realizado y el proceso seguido; en segundo lugar, recoger la percepción de los estudiantes sobre la técnica empleada; el tercero, recabar información del alumno sobre el papel de los compañeros en el aprendizaje de la materia y la autonomía del grupo en la resolución de problemas (véase tabla 1). Por último, considerar los resultados obtenidos y analizar si la situación de partida ha mejorado - lo que implicaría seguir con la misma planificación — o se modifica y se diseña un nuevo ciclo.

Tabla 1. Preguntas realizadas a los estudiantes

\begin{tabular}{ll}
\hline \multicolumn{1}{c}{$\begin{array}{c}\text { PREGUNTAS ABIERTAS } \\
\text { (Análisis cualitativo) }\end{array}$} & $\begin{array}{l}\text { PREGUNTAS CERRADAS } \\
\text { (Análisis cuantitativo) }\end{array}$ \\
\hline - Menciona algo que has aprendido de tus & - Número de miembros en tu \\
compañeros de grupo que probablemen- & $\begin{array}{l}\text { equipo } \\
\text { te no hubieras aprendido por tu cuenta. }\end{array}$ ¿Cuántos trabajaron adecua- \\
- Indica los puntos fuertes y débiles de la & damente? \\
experiencia. & \\
¿Habéis tenido algún problema? ¿Cómo & \\
lo habéis resuelto? & \\
\hline
\end{tabular}

Como muestra la tabla 1, el cuestionario empleado se centra en dos partes: la primera ofrece cuestiones específicas mediante preguntas abiertas, no encaminadas a la cuantificación, cuyo objetivo es que el encuestado aporte aspectos que considera importantes en relación con la experiencia y se le solicita que describa con la mayor precisión posible lo que experimenta y cómo actúa; la segunda propone preguntas cerradas cuantificables.

Esta experiencia se lleva a cabo en dos grupos diferenciados. Por una parte, el grupo de inglés como lengua extranjera (ILE), constituido por 32 estudiantes de nacionalidades diversas (españoles, alemanes, checos, húngaros, italianos, polacos y turcos), en el último curso de ingeniería y con un nivel heterogéneo de lengua extranjera, distribuidos en grupos de entre 3 y 5 miembros cada uno. Por otra, el grupo de español como lengua materna formado por 22 estudiantes de español como lengua materna (ELM), de últimos cursos de ingeniería y tres estudiantes Erasmus, agrupados en equipos de entre 3 y 4 participantes por grupo. 


\section{Método}

De acuerdo con la estructura cíclica de la investigación-acción se planifica la implementación de la técnica de aprendizaje colaborativo del puzle de Aronson y la recogida de datos de ambos grupos en base a varios objetivos de aprendizaje fundamentales. Por una parte, la mejora de las destrezas comunicativas tanto en lengua extranjera como en lengua materna - que no son motivo de análisis en este estudio- a través del trabajo en equipo; por otra, el aprendizaje individual y colaborativo que les ayude a ser aprendices autónomos tanto desde el punto de vista lingüístico como de los contenidos propios del proyecto elegido por cada grupo. La acción se desarrolla tanto en horas presenciales de clase como fuera de ellas durante tres semanas; la observación se lleva a cabo de manera directa en el aula y en tutorías.

Como instrumento de investigación se elabora un cuestionario (véase tabla 1) compuesto de dos preguntas cerradas que limitan la respuesta de los sujetos pero que nos proporcionan datos concretos sobre lo que se quiere saber: número de miembros del grupo e información de los que han trabajado adecuadamente (datos estadísticos); junto a tres preguntas abiertas que investigan actitudes y aprendizaje que nos proporcionen información sobre el cambio que se haya podido producir al introducir el puzle y que se analizan cualitativamente.

Para analizar los resultados se efectúa, en primer lugar, un análisis cuantitativo de las respuestas a las dos preguntas relacionadas con el número de participantes asignado a cada grupo de trabajo y el número real de compañeros que ha llevado a cabo la tarea al completo; el objetivo es averiguar si existen diferencias significativas entre el grupo ILE y el ELM respecto al número de miembros que no ha trabajado en la multitarea adecuadamente, según sus compañeros de equipo. Para ello, se comparan las medias de ambos grupos como muestras independientes a través de la T-student; prueba diseñada para muestras pequeñas, inferiores a 30 elementos como en este caso, y en cuya aplicación se cumplen todas las condiciones que este test exige.

El estudio se completa, por una parte, con el análisis cualitativo de las respuestas a tres preguntas que recaban las percepciones de los participantes respecto a los puntos fuertes y débiles de la experiencia; por otra, con lo que consideran que han aprendido de sus compañeros de equipo gracias a la técnica, así como a las dificultades que han tenido y 
su modo de resolverlas. Por último, se comparan los resultados obtenidos en ambos grupos.

El análisis cualitativo de este estudio está basado en la teoría fundamentada de Glaser y Strauss (1967, ed. 2009) sobre las opiniones individuales vertidas en las tres preguntas abiertas. Se basa en una guía flexible y sistemática de recogida y análisis de datos con objeto de construir una teoría basada en ellos; esos datos conforman los cimientos de la teoría y su análisis general las categorías teóricas o conceptos sobre los que se construye. Para la codificación de los datos se ha utilizado la aplicación Atlas.ti v. 5.2. (Casasempere, 2009).

\section{Resultados}

La tabla 2 ofrece el resumen estadístico de ambos grupos, de acuerdo con las opiniones de los propios compañeros. De una muestra de 32 participantes en el grupo ILE, una media de 0,343 no ha trabajado adecuadamente (de acuerdo con una de las preguntas cerradas del cuestionario, tabla 1); en el grupo ELM, con una muestra de 25 integrantes, una media 0,24 tampoco lo ha hecho. Sobre esas medias se realiza la comparación como muestras independientes a través de la T-student.

Tabla 2. Resumen estadístico

\begin{tabular}{lll}
\hline & ILE & ELM \\
\hline Muestra & 32 & 25 \\
Media & 0,34375 & 0,24 \\
Desviación estándar & 0,653002 & 0,522813 \\
Mínimo & 0,0 & 0,0 \\
Máximo & 2,0 & 2,0 \\
\hline
\end{tabular}

En el resumen estadístico el valor mínimo recoge el número de estudiantes mínimo que no ha trabajado adecuadamente en las dos muestras (0 en ambos casos) y máximo ( 2 miembros por grupo en ambos casos). Al aplicar la prueba T-student sobre la comparación de medias de ambas muestras se obtiene un valor de $-0,648153$, lo que señala que no existen diferencias estadísticamente significativas entre las medias de las dos muestras de datos para un nivel de confianza del $95 \%$. 
El gráfico 1 que el paquete estadístico ${ }^{6}$ realiza junto con la T-student ofrece en su columna de la izquierda el número total de estudiantes en el grupo ILE (parte inferior del gráfico) y ELM (parte superior) que ha trabajado adecuadamente sobre el total de ambas muestras. La columna del centro señala el número de estudiantes que considera que uno de sus compañeros de grupo no ha trabajado adecuadamente mientras que la de la derecha ofrece el número de aquellos que considera que en su grupo ha habido dos compañeros que no han trabajado a lo largo del proceso.

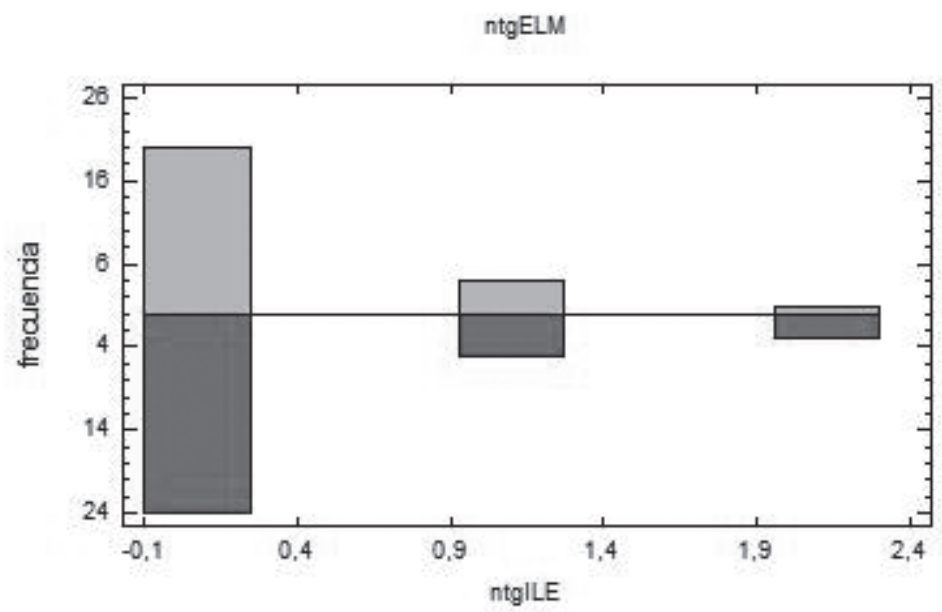

\section{Gráfico 1. Número de participantes que no ha trabajado adecuadamente en ILE y en ELM}

Los dos subapartados siguientes ofrecen los resultados del análisis cualitativo sobre los puntos fuertes y débiles de la experiencia, lo que consideran que han aprendido de sus compañeros de equipo gracias a ella, y cómo han resuelto los posibles problemas.

\section{Grupo ILE}

Siguiendo el procedimiento de análisis surge la teoría que a continuación se propone y que está fundamentada en las palabras y el sentir de los estudiantes de inglés como lengua extranjera. De acuerdo con las respuestas obtenidas, la experiencia con el puzle de Aronson parece

6 Statgraphics Centurion XV, v. II 
haberles supuesto un entrenamiento para la vida real y profesional. Esta teoría se basa en dos grandes categorías extraídas de sus respuestas y que ayudan a comprender el proceso que han vivido: el contenido de la experiencia y la manera de llevarla a cabo (las dos categorías en torno a las cuales gira la experiencia para este grupo). Se incluyen citas textuales entre comillas seguidas de un número identificativo.

El haber podido elegir el tema de su proyecto de trabajo en grupo lo consideran una buena idea que ha hecho que "aumente su interés e implicación" (p12). En uno de los grupos, no obstante, el profesor no ha detectado su mal funcionamiento debido, en palabras de los participantes, a la "falta de interés por el tema elegido" (p3) lo que se ha traducido en "desinterés y en no emplear el tiempo necesario para realizar la tarea individual y grupal" (p4).

Sin embargo, todos los demás consideran que han realizado una "tarea útil" (p20) que, además de aportarles conocimientos específicos, "les ha ofrecido la posibilidad de mejorar sus destrezas comunicativas" (p17) en lengua extranjera al tener que "hablar en público y en inglés entre sus compañeros" (p24), además de servirles "para desarrollar las relaciones sociales dentro y fuera del propio grupo de trabajo" (p32). El que cada grupo contara con niveles heterogéneos de competencia comunicativa así como con miembros de diferentes nacionalidades lo consideran una "ventaja para aprender" (p30) y para "ser más pacientes los unos con los otros por proceder de culturas distintas" (p29); algo que con bastante probabilidad van a tener que afrontar en su futuro como ingenieros. Consideran que "han aprendido los contenidos objeto del trabajo en grupo" (p28) aunque, para algunos, "se debería aumentar la interacción entre grupos" (p16), si bien no indican cómo.

Como puntos débiles se encuentran dos categorías, el tiempo que el puzle requiere y la falta de solidaridad de alguno de los participantes. Estiman que se trata de "una técnica activa que consume tiempo" (p30) y que "precisa atención" (p2), especialmente si uno tiene "falta de confianza en sí mismo o en el tema de trabajo elegido por el grupo" (p5) "al tener que dedicarle más tiempo y esfuerzo para paliar esa inseguridad" (p32). Los diferentes orígenes de los participantes han contribuido, para la gran mayoría, a que la experiencia fuera más enriquecedora. Sin embargo, en aquellos casos aislados en los que algún miembro del grupo no ha desempeñado sus funciones de manera adecuada, se ha traducido en "falta de comunicación" (p4) y "malentendidos" (p7), de manera 
que los diferentes acentos y niveles de lengua se han convertido en un obstáculo más que en una ventaja para aprender. Compartir ideas entre los miembros de un grupo parece ser para algunos participantes más complejo "con más de tres personas por grupo" (p6) cuando se trata de "trabajar fuera de las horas de clase" (p7); algo dificultoso para algún equipo, salvo si el tema del proyecto elegido les resulta interesante.

Lo que consideran que han aprendido de los demás miembros de su equipo se traduce en dos categorías: trabajar en equipo y los contenidos del proyecto. La primera les ha proporcionado "otros puntos de vista y la posibilidad de hacer las cosas de una manera diferente" (p11) a como suelen hacerlas además de "ayudar[les] en la comprensión y expresión oral" (p8). Respecto a los contenidos de su proyecto, reconocen "la importancia del lenguaje corporal" (p5) en la comunicación, "la estructura" (p3) de su proyecto, la importancia de utilizar la "gramática correctamente" (p9), unida a un "vocabulario y expresiones específicas relacionadas con el tema trabajado" (p30).

Sólo un grupo manifiesta haber tenido conflictos que resolver; el resto afirma haber trabajado "como verdaderos equipos" (p13), con las discusiones y acuerdos necesarios "para poder finalizar la tarea con éxito dentro del plazo previsto" (p32). En ese único caso conflictivo los miembros del equipo afirman "no haberse llevado bien" (p5) y achacan el problema al hecho de ser de diferentes países (y culturas) y "no haber sido capaces de establecer una comunicación adecuada entre ellos" (p4).

Por último, se aprecia lo que otro grupo no tilda de problema propiamente pero admite que les ha causado cierto trastorno: "el que todos sus miembros no estuvieran siempre presentes para trabajar dentro y fuera del aula" (p16).

\section{Grupo ELM}

Para los estudiantes de español como lengua materna la experiencia parece haberles descubierto las posibilidades que el trabajo colaborativo puede aportarles frente a otras técnicas de enseñanza-aprendizaje. Esta teoría se basa en dos categorías: lo que les ha aportado el trabajo en equipo y el uso de una metodología activa como es el puzle frente a la lección magistral, técnica a la que parecen estar más acostumbrados. Consideran que el trabajo en equipo, tras un trabajo individual previo, les ha ayudado a "aprender con mayor facilidad" (p1) sobre el tema 
tratado al tiempo que, al tener que poner en común pensamientos y conocimientos específicos sobre él, les ha aportado "nuevas ideas a través del diálogo con sus compañeros" (p20); lo que, en su opinión, ha traído consigo la "posibilidad de socializar en el aula" (p4) y de "conocer mejor a sus compañeros de asignatura" (p5).

Por lo general piensan que la técnica ha contribuido a crear "una atmósfera más motivadora que la propia lección magistral" (p10). Además de ofrecerles "ejemplos de la vida real" (p8) y ayudarles a "adquirir conocimientos específicos" (p10), el hecho de tener que instruir y aprender de sus compañeros de equipo lo han entendido como una manera de "aprovechar el tiempo" (p23) y de "aprender más" (p11); aspecto que les ha ayudado a "mejorar la comunicación oral al tener que resumir y sintetizar las ideas principales a sus compañeros" (p6) y asegurarse de que han aprendido esos contenidos. Valoran, como metodología activa que es, el hecho de que tengan que "hacer cosas, no sólo leer" (p12), como contrapunto de lo que parece que suelen hacer habitualmente en sus estudios.

Encuentran pocos puntos débiles a la experiencia con la técnica del puzle; por una parte, acusan una "falta de tiempo" (p22) especialmente durante la comprensión de la parte de la unidad explicada por sus propios compañeros de equipo como expertos de ese contenido y, por otra, la "falta de asistencia de los miembros" (p8) de algunos grupos; algo que les ha hecho reflexionar sobre lo que afecta a un equipo no sólo la falta de asistencia sino también "la importancia que tiene para todos el cumplimiento de las tareas individuales" (p14) para la consecución de la meta común. Unas reflexiones que sin duda les ayudarán en sus estudios y futuro profesional.

La mayoría de los participantes da ejemplos concretos de los contenidos que han aprendido a través de las explicaciones de sus compañeros de equipo, además de creer que gracias a éstos (verdaderos docentes en esta parte del proceso) han "sintetizado mejor los contenidos" (p24); algún caso concreto admite no saber qué contestar a este respecto y llama la atención la reflexión que se hace al darse cuenta de aquello "que no hicieron y debieron haber hecho durante la experiencia" (p18): como no se presentaron entre ellos al inicio de la actividad, "acabaron por no saber ni los nombres de los compañeros de grupo" (p16), una reflexión que les ayudará a cambiar de actitud en las ocasiones en las que el trabajo en equipo sea la estrategia a emplear. 
No se han presentado conflictos dentro de los equipos de trabajo, si bien se detecta la falta de algún miembro en dos de ellos; sin embargo, no parece que adoptaron ninguna medida al respecto y continuaron los demás participantes con la tarea encomendada.

\section{Conclusiones}

Pese a tratarse de asignaturas de lengua diferentes, con una composición de equipos distinta (aunque con un problema común), observamos que comparten rasgos que giran en torno al sentimiento de sus participantes a lo largo del proceso y al reconocimiento expreso de sus debilidades y puntos fuertes. Mientras los unos consideran la experiencia como un entrenamiento para la vida real con competencias lingüísticas y procedencias diferentes, los otros comparan la técnica, y lo que les ha aportado, con la estrategia más tradicional con la que conviven diariamente en el aula universitaria; lo que, en el fondo, parece subyacer es que han vivido un proceso de trabajo con el que no están demasiado familiarizados al no haberse puesto en práctica todavía en demasiadas asignaturas de sus carreras pero del que aprecian los resultados obtenidos.

En el análisis de resultados se observa, sin embargo, una diferencia fundamental entre un grupo y otro: el que cada uno pudiera elegir el tema sobre el que trabajar a lo largo del proceso o el que hubiera un único tema para todos los equipos, tal y como ha sido en el caso de ILE y ELM, respectivamente.

No obstante, respecto a los puntos fuertes se observan similitudes en una gran parte de sus respuestas; tal es el hecho, por una parte, de que en ambos grupos los estudiantes perciban la experiencia como un entrenamiento para su futura labor profesional (ILE) que les ha ofrecido ejemplos de la vida real (ELM). Por otra, todos opinan que la técnica del puzle les ha ayudado a mejorar sus destrezas comunicativas en lengua extranjera y materna.

Consideran como puntos débiles el tiempo que la técnica requiere, especialmente si los estudiantes están poco acostumbrados a las metodologías activas como parece ser el caso, y la problemática que en todo grupo de trabajo se puede plantear cuando alguno de sus miembros no asiste a las reuniones de equipo (dentro y/o fuera del aula). Todo ello se convierte, en nuestra opinión, en un aprendizaje valioso cuando son los 
propios estudiantes quienes reparan en la importancia que tiene la asistencia y el cumplimiento de las tareas individuales para el bien común del grupo.

Durante las tres semanas que ha durado la actividad, no se han presentado conflictos excepto en uno de los equipos debido, según apuntan, a la diferencia cultural y de procedencia de sus miembros y a la incapacidad de establecer una comunicación fluida entre ellos; algo que para los demás grupos en su misma situación se ha considerado una ventaja para aprender.

Desde el punto de vista cuantitativo, la falta de diferencias estadísticamente significativas entre la media de alumnos que no ha trabajado adecuadamente según sus compañeros en ILE y en ELM señala, asimismo, que las características específicas de cada gran grupo no han interferido en el desarrollo de la actividad.

La finalización de todas las tareas muestra la satisfacción y la motivación de los grupos durante todo el proceso. El único obstáculo encontrado ha sido el referido a las ausencias, que como ya hemos indicado, se ha resuelto dentro de los respectivos equipos; este pequeño inconveniente lo han convertido en una reflexión positiva al comprender cómo afecta negativamente este tipo de comportamiento, tanto de forma personal como en la labor del equipo.

Estos resultados nos motivan a seguir apostando por tareas de trabajo en equipo con metodologías activas que resulten significativas para nuestros estudiantes y que les acerquen, una vez más, a su entorno académico y profesional. Hemos podido observar como, junto con conocimientos relacionados con las asignaturas, adquieren competencias comunicativas y de trabajo en grupo que se le van a exigir en su profesión, además de aprender a valorar la productividad de su grupo, los puntos fuertes y aquéllos que precisan mejora. En definitiva, el puzle de Aronson se muestra como una estrategia apropiada para conseguir determinados objetivos de aprendizaje específicos y transversales que ha de complementarse con otros métodos, técnicas y estrategias activas en función de los objetivos de enseñanza-aprendizaje que se persigan.

Los resultados globales evidencian una mejoría respecto a la situación de partida. Recordemos que en ambos grupos nos encontramos con estudiantes que trabajaban de manera ineficiente, con aportaciones dispares, tareas sin concluir y ausencias habituales. Si perseguíamos promover la responsabilidad de los miembros de los grupos, se constata 
cómo la tarea implementada con el puzle de Aronson ha fomentado su interdependencia positiva; algo que ha sido posible gracias al diseño de una actividad en la que los estudiantes han experimentado que el éxito de cada uno depende, en gran medida, de la labor de todos. Puesto que no todos los grupos trabajan a la misma velocidad, el haber concretado para cada una de las fases un tiempo límite ha servido de ayuda a los participantes para vigilar su progreso.

Para solucionar el problema de las ausencias, los docentes han de recordar a los grupos que se evalúa tanto el proceso como el producto final, tal y como ha sucedido en la experiencia descrita; de ahí que tal inconveniente lo hayan resuelto a través de unas reglas de funcionamiento que ellos mismos han acordado. Entre las soluciones aportadas han contemplado la utilización de medios alternativos de comunicación, esencialmente del correo electrónico y del chat; un comportamiento que da claros indicios de que han conseguido trabajar como grupos autónomos, que gestionan su trabajo para conseguir el objetivo común. Consideramos que para lograrlo la clave está en que todos sientan la pertenencia a un grupo y la necesidad de colaboración entre sus miembros.

La implementación de la técnica del puzle ha modificado de manera significativa todos los problemas de partida; por ello no se ha considerado necesario un nuevo ciclo de investigación o una modificación del plan ejecutado. Como investigaciones futuras cabe plantearse, entre otras, la inclusión de la observación no participante en las experiencias, así como la comparación de resultados con otra metodología de trabajo en grupo como es el aprendizaje basado en problemas o en proyectos en la que, además de la técnica, varíe el número de estudiantes por grupo pero se mantengan los objetivos; con estos cambios se podrá observar si se modifican las percepciones de los estudiantes y el número de participantes por grupo que trabaja adecuadamente.

\section{Referencias bibliográficas}

ABET. Acreditation Board of Engineering and Technology (2000). Criteria for accredting engineering programas. Baltimore, MD: Anthor.

Aranguren, G. (2007). La investigación-acción como estrategia de intervención y formación del docente en su rol de investigador. Revista de Pedagogía, 28(82), 173-195.

Barkley, E.F., Cross, K. P. y Major, C. H. (2009). Técnicas de aprendizaje colaborativo. Madrid: Ediciones Morata, S.L. 
Beverly, J. (1993). Teacher-as-researcher. ERIC Digest. Obtenido de http://www.ericdigests.org/1993/researcher.htm

Bartolome Pina, M. (1992). Investigación cualitativa en educación: ¿comprender o trasformar? Revista de Investigación Educativa, 20,7- 36.

Bruffee, K.A. (1993). Collaborative learning: Higher education, interdependence, and the authority of knowledge. Baltimore, MD: Johns Hopkins University Press.

Brydon-Miller, M., Greenwood, D. y Maguire, P. (2003). Why Action Research? Action Research, 1(1), 9-28.

Burns, A. (2010). Doing Action Research in English Language Teaching. A guide for Practitioners. New York: Routledge.

Carr, W. (1989). Action Research: Ten years on. Journal of Curriculum Studies, 21 (1), 85-90.

Carr, L. y Kemmis, S. (1986). Becoming critical: Knowing through action research. London: Croom Helm.

Casals, A., Vilar, M. y Ayats, J. (2008). La investigación-acción colaborativa: reflexiones metodológicas a partir de su aplicación en un proyecto de música y lengua. Revista Electrónica Complutense de Investigación en Educación Musical, 5 (4). Obtenido de http://www.ucm.es/info/reciem/v5n4.pdf

Casasempere, A. (2009). Inmigración y Educación. Curso Atlas.ti. Obtenido de http:// www.popdeporte.uma.es/pdf/Curso_ATLAS.ti_2008\%20_mayo_.pdf

Cochran-Smith, M. y Little, S. (1993). Research on Teaching and Teacher Research: The Issues that Divide. Educational Researcher, 19 (2), 2-11.

Charmaz, K. (2006). Constructing Grounded Theory. A Practical Guide through Qualitative Analysis. London: SAGE

Cuseo, J.B. (1992) Cooperative learning: A pedagogy for diversity. Cooperative learning \& College Teaching, 3(1), 2-6.

Dansereau, D.F. (1988). Cooperative estrategies. En C. Weistein. (Ed.), Learning and study strategies: Issues in assessment, instruction and evaluation. (pp. 209-239). New York: Academic Press.

Echazarreta, C., Prados, F. Poch, J. y Soler, J. (2009). La competencia "el trabajo colaborativo": una oportunidad para incorporar las TIC en la didáctica universitaria. Descripción de la experiencia con la plataforma ACME (EdG). UOC.Papers. Revista sobre la sociedad del conocimiento, 1-11 Obtenido de

http://www.uoc.edu/uocpapers/8/dt/esp/echazarreta_prados_poch_soler.pdf

Elliot, J. (1990, ed. 2010). La investigación-acción en educación. Madrid: Ediciones Morata, S.L.

Elliot, J. (1993). El cambio educativo desde la investigación-acción. Madrid: Morata.

Englert, C. S., Berry, R., y Dunsmore, K. (2001). A case study of the apprenticeship process; another perspective on the apprentice and the scaffolding metaphor. Journal of Learning Disabilities, 34 (2), 152-171.

Flannery, J.L. (1994). Teacher as co-conspirator: Knowledge and authority in collaborative learning. En K Bosworth y S, Hamilton (Eds.), Collaborative learning: Underlying process and effective techniques, 15-23. New Directions for Teaching and Learning, 59, San Francisco: Jossey-Bass. 
Investigación-acción para conseguir grupos colaborativos eficaces

María José Labrador-Piquer y María Ángeles Andreu-Andrés

Ghaith, G. (2004). Correlates of the implementation of the STAD cooperative learning method in the English as a foreign language classroom. International Journal of Bilingual Education and Bilingualism, 7 (4), 279-294.

Glaser, B.G. y Strauss, A. (1967, ed. 2009). Discovery of Grounded Theory. Strategies for Qualitative Research. New Jersey: Sociology Press.

Goetz, J. L. y Le Compte, M. D. (1984). Ethnography and Qualitative Design in Eduactional Research. New York: Academic Press.

Gocer, A. (2010). A comparative research on the effectivity of cooperative learning method and jigsaw technique on teaching literary genres. Educational Research and Reviews, 5 (8), 439-445.

Grasha, A. (2002). Teaching with Style. Pittsburgh, PA: Alliance Publishers. Obtenido de http://www.ius.edu/ilte/pdf/teaching_with_style.pdf

Hammersley, M. y Atkinson, P. (1983). Ethnography: Principles and Practice. Londres: Tavistock Publications.

Holter, I.M., y Schwartz-Barcott, D. (1993). Action Research: What is it? How has it been used and how can it be used in nursing? Journal of Advanced Nursing, 18 (2), 298304.

Jiménez-Domínguez, B. (1994). Investigación ante acción participante: una dimensión desconocida. En M. Montero (Ed.), Psicología Social Comunitaria: Teoría, método y experiencia (pp. 103-137). Guadalajara: Universidad de Guadalajara.

Johnson, D.W., Johnson, R.T. y Smith, K.A. (1991, ed. 2009). Active Learning: Cooperation in the College Classroom. Edina, Minnesota: Interaction Book Company.

Kemmis, S. y McTaggart, R. (1988). The action research planner. Geelong: Deakin University Press.

Labrador, $M^{a}$ J. (2011). Aprendizaje colaborativo a través de problemas. Revista Foro Anual de Profesores de ELE, Foro VII, Universitat de València. Obtenido de http:// www.uv.es/foroele/foro7/articulos/Labrador.pdf

Labrador, Ma J., Morote, P. y Mula, A. (2009). Hablar, leer y escribir mediante la publicidad. Prácticas de lectura y escritura. Brasil: Universidade de Passo Fundo: 139-164.

Latorre, A. (2003, ed. 2010). La investigación-acción. Conocer y cambiar la práctica educativa. Barcelona: Graó.

Lewin, K. (1946). Action Research and Minority Problems. Journal of Social Issues, 2 (4), 34-46.

López Górriz, I. (1993). La investigación-acción como metodología de teorización y formación del profesor desde su práctica. Revista de Investigación Educativa, 6,71-92.

McKernan, J. (1996). Teaching the foundations of education: values, proceduralneutrality and the action research seminar. En Powers, E. (Ed.), 1996 Year of the South Atlantic Philosophy of Education Society. Greenville, N.C.: East Carolina University.

Masters, J. (1995). The History of Action Research, en I. Hughes (Ed.) Action Research Electronic Reader, The University of Sydney, Obtenido de http://www.behs.cchs.usyd.edu.au/arow/Reader/rmasters.htm

Matthews, R.S. (1996). Collaborative learning: Creating knowledge with students. En R.J. Menges, M. Weimer y Assoc. (Eds.), Teaching on solid ground: Using scholarship to improve practice (pp. 101-124). San Francisco: Jossey-Bass. 
Investigación-acción para conseguir grupos colaborativos eficaces

María José Labrador-Piquer y María Ángeles Andreu-Andrés

McKernan, J. (1991, ed. 2010). Curriculum Action Research. A Handbook of Methods and Resources for the Reflective Practitioner. London: Kogan Page.

Passow, H.J. (2012). Which ABET Competencies do Engineering Graduates find most important in their work? Journal of Engineering Education, 101 (1), 95-118.

Pérez-Sánchez, A., Poveda-Serra, P. y Gilar-Corbí. R. (2010). Efectos del aprendizaje colaborativo en el uso de estrategias de afrontamiento. Revista Latinoamericana de Psicología, 42(3), 481-492.

Quintero, J., Zuluaga, C. y López, M. (2003). La investigación-acción mejora la planeación de clases en lengua extranjera. Ínkala, revista de lenguaje y cultura, 8 (14), 39-56 Obtenido de http://revinut.udea.edu.co/index.php/ikala/article/viewFile/3178/2942

Rincón Igea, D. y Rincón Igea, B. (2000). Revisión, planificación y aplicación de mejoras. Revista Interuniversitaria del Profesorado, 39, 51-73.

Schön, D.A. (1983). The reflective practitioner: How professionals think in action. New York: Basic Books.

Schön, D. A. (1987). Educating the Reflective Practitioner. San Francisco: Jossey-Bass Publishers.

Sim, J. y Radloff, A. (2008). Enhancing reflective practice through online learning: impact on clinical practice. Biomedical Imaging and Intervention Journal, 4(1), e8 Obtenido de http://www.biij.org/2008/1/e8/e8.pdf

Slavin, R.E. (1990). Cooperative Learning: Theory, Research and Practice. Boston: Ally and Bacon.

Stenhouse, L. (2003). Investigación y desarrollo del currículum. Madrid: Ediciones Morata, S.L.

Stenhouse, L. (2009). La investigación como base de la enseñanza. Madrid: Ediciones Morata, S.L.

Traver, J.A. y García, R. (2006). La técnica puzle de Aronson como herramienta para desarrollar la competencia compromiso ético y la solidaridad en la enseñanza universitaria. Revista Iberoamericana de Educación, 40 (4), 1-9.

Winter, R. (1987, ed. 2009). Action Research and the Nature of Social Inquiry: Professional Innovation and Educational Work. Averbury, England: Aldershot.

Woods, P. (1986). La escuela por dentro: La etnografía en la investigación educativa. Barcelona: Paidós. 
